Textures and Microstructures, 1988, Vol. 7, pp. 303-316

Reprints available directly from the publisher

Photocopying permitted by license only

(C) 1988 Gordon and Breach Science Publishers Inc.

Printed in the United Kingdom

\title{
Piezoelectric Pole Figure of a Vein Quartz Sample
}

\author{
M. M. GHOMSHEI, B. B. NAROD, T. L. TEMPLETON, ${ }^{*}$ A. S. \\ ARROTT,* and R. D. RUSSELL \\ Department of Geophysics and Astronomy, University of British Columbia, \\ Vancouver B. C. Canada, V6T 1W5
}

(Received October 20, 1987)

Longitudinal piezoelectric coefficients have been measured on cubic samples and from 150 different orientations on a spherical sample of coarse-grained vein quartz. The results are consistent with the presence of a piezoelectric fabric. The piezoelectric pole figure of the studied spherical sample demonstrates three distinct patterns of alternatively positive and negative signs defining one strong and two weak electric dipoles in the fabric. The magnitude of the piezoelectric effect along the major maximum is as large as $7 \%$ of the theoretical effect in a single crystal and is considerably higher than the expected statistical effect. Preferred orientation of the $a$-axes, was independently confirmed by subjecting the same spherical sample to neutron diffraction analysis. The location of nonpolar $a$-axes maximum determined by neutron diffraction analysis agrees with the piezoelectric maximum which is theoretically related to the polar preferred orientation of a-axes. The piezoelectric effect and neutron diffraction are both volume events and ensure reliable statistics for analysis of coarse-grained quartz aggregates where surface events (such as X-ray diffraction) may suffer poor statistics.

KEY WORDS: Piezoelectric fabric, Vein quartz, Neutron diffraction, Charge pole figures, Texture pole figures.

\section{INTRODUCTION}

Piezoelectricity is a characteristic of certain crystals (especially dielectrics) which lack a centre of symmetry. It is a third rank tensor

\footnotetext{
* Department of Physics, Simon Fraser University, Burnaby B. C. Canada, V5A 1S6
} 
defined by the general equation:

$$
P_{i}=D_{i j k} s_{j k}
$$

Where $P$ is the polarization (vector), $s$ is the second rank stress, and $D$ (the constant of proportionality) is the third rank piezoelectric moduli tensor (Nye, 1957; Cady, 1964).

The piezoelectric moduli of quartz are demonstrated in the following matrix:

\begin{tabular}{ccccccc}
\multicolumn{4}{c}{ longitudinal } \\
stress & \multicolumn{3}{c}{ shear stress } \\
& $s_{1}$ & $s_{2}$ & $s_{3}$ & $s_{4}$ & $s_{5}$ & $s_{6}$ \\
$P_{1}$ & $d_{11}$ & $-d_{11}$ & 0 & $d_{14}$ & 0 & 0 \\
$P_{2}$ & 0 & 0 & 0 & 0 & $-d_{14}$ & $-2 d_{11}$ \\
$P_{3}$ & 0 & 0 & 0 & 0 & 0 & 0
\end{tabular}

with reference to an orthogonal system in which one of the $a$-axes of quartz coincides with $X_{1}$ and the optical axis with $X_{3}$. This matrix demonstrates that longitudinal stress produces polarization only along the $a$-axes and the $c$-axis can never be polarized regardless of direction and nature of stress.

Rocks bearing piezoelectric minerals have been found to be piezoelectric, either due to statistical fluctuation and the effect of large uncompensated grains (Tuck et al. 1977) or due to polar preferred orientation (Parkhomenko, 1971, Wellmer, 1970 \& 1971, Bishop, 1981a, 1981b; Barid and Kennan, 1985). The statistical effect is inversely proportional to the square root of the number of grains and would tend to zero with decreasing grain size. The statistical effect is inherently independent of sample orientation and its value is a function of grain size. The statistical effect for polar events (having positive and negative values) produces, however, an incoherent background with zero expectation value. It means, when analysing cubic samples cut from a large specimen (traditional practice), the measured polarization on similar faces may vary in sign and magnitude, oscillating about zero average, when no fabric effect is present.

The fabric-related effect (also called true piezoelectricity), if present, is orientation-dependent and may be very small along the axes of the arbitrarily cut cubic samples. In this situation the fabric 
may be detected only when a statistically large number of oriented cubic samples are analysed and the mean value of the measurements on similar faces tends to a non-zero value related to the fabric effect (fabric inhomogeneities and experimental errors being neglected). It is therefore a difficult task to distinguish the true piezoelectricity when the statistical effect is not considerably lower than the fabric effect. For aggregates with a strong fabric effect there is a good chance that, for at least along one of the axes of the cubic samples under study, the true piezoelectricity dominates the statistical effect. In this case it may be possible to distinguish the fabric effect by analysing only a small number of samples.

The search for natural piezoelectric fabrics has been carrried out for different purposes. A considerable volume of literature on this subject concerns the application of the effect in seismoelectric exploration and interpretation of some radio emissions associated with earthquakes (e.g. Sobolev et al. 1980, 1982, 1984, Nitsan 1977, Warwick et al. 1982). Some investigators (e.g. Bishop, 1981) have shown interest in the application of the effect in petrofabric analysis, while others involved in basic research reiterated the concept of paleoelectrification as the important implication of the natural piezoelectric fabrics (Tuck et al. 1977).

Previous methods for determining the piezoelectric fabric of a rock have been based on the measurement of nine apparent longitudinal piezoelectric moduli of cubic samples (no shearing stresses applied). These measurements are assumed to approximate the rotated piezoelectric matrix of one of the perfect theoretical piezoelectric fabrics multiplied by a dilution factor (Wellmer, 1970; Parkhomenko, 1971; Bishop, 1981). The model-fitting approach is attractive as it involves only nine measurements. However, due to the complexity of natural fabrics the experimental data usually do not fit reliably to any single theoretical model.

Bishop (1981b), provided evidence for the existence of fabric related piezoelectricity in some mylonitic quartz aggregates. He measured effects considerably larger than the expected statistical effect. He also provided further evidence for existence of a fabric related effect by observing repeatability of measurements in several cubic samples shaped from a single large specimen.

Strong piezoelectric effects in some coarse-grained aggregates have been reported by Soviet investigators (Parkhomenko, 1971). 
Coarse-grained aggregates may, however, demonstrate strong piezoelectricity due to the statistical effect and the effect of large uncompensated grains (Tuck et al. 1977) which may overshadow the fabric related effect, if present.

While the traditional practice of analysing cubic samples has proven successful in predicting the symmetry and orientation of the piezoelectric fabric of some mylonitic quartz aggregates (Bishop, 1981b), the present work is an attempt towards a more objective and complete assessment of the piezoelectric effect by systematic scanning of a spherical sample. Although the analytical procedure is, at this stage, time consuming, it is rewarding because the obtained experimental data are self-sufficient for diagnosis and description of the fabric-related effect in a single sample, without recourse to model fitting and supplementary fabric analysis. We have also analysed the same spherical sample by neutron diffraction, which is a volume event similar to piezoelectricity, for comparison of the $a$-axes orientation with the measured piezoelectric effect.

We chose to study vein quartz because of its large piezoelectric effect which can possibly be applied in field seismoelectric exploration. The samples have been collected from the site of a field experiment where strong electric signals were detected from the vicinity of the quartz vein after detonation of an explosive charge. Questions arose as to whether the quartz aggregate possesses a true piezoelectricity and, if so, what is the orientation of the maximum effect?

\section{PIEZOELECTRIC ANALYSIS}

\section{(A) Instrumentation}

The laboratory apparatus for the measurement of piezoelectric signals (Figure 1) has been designed to simulate acoustic shocks which would be produced by explosions in the vicinity of quartz veins in field experiments. The impulses readily produced stresses in the frequency range of interest, up to several kilohertz.

The apparatus is based on a two metre optical bench which 


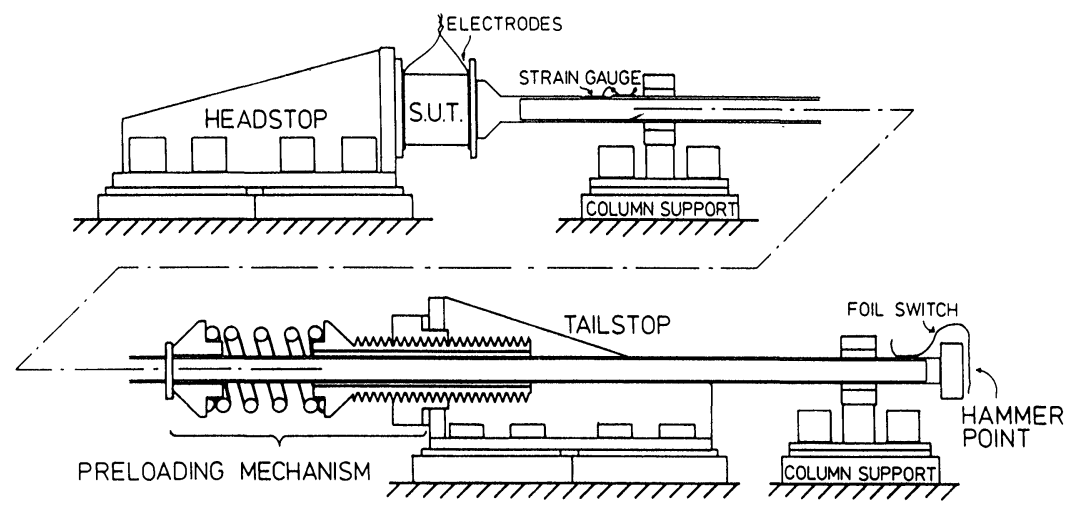

Figure 1 Schematical cross-section of experimental apparatus for the measurement of piezoelectric signals.

weighs approximately $100 \mathrm{~kg}$. The bench acts as an anchor for the experiment and minimizes the unwanted vibrational effects.

The sample under test and the impacting column are supported between two movable stops, a rigid headstop and a spring loaded tailstop. The spring loading acts to pretension the system in order to minimize microphonic effects at the electrodes. It also acts to pass all the stress applied at the hammer point through to the sample.

The impacting column consists of a stainless steel tube $9 \mathrm{~mm}$ O.D. and $1 \mathrm{~mm}$ wall thickness. A strain gauge placed on the column near the sample provides a measure of the axial stress. Additional column supports and damping mechanisms, not shown in the figure, minimize the unwanted flexural and axial oscillations. Added mass on the column has been kept to a minimum to reduce the required amount of additional damping. When struck with a hand operated rubber mallet, the apparatus produces a nearly critically damped impulse, band-limited to a range below $500 \mathrm{~Hz}$. Longer columns would be required to lower the frequency range.

For a crystal with piezoelectric modulus $d_{11}=2 \mathrm{pC} / \mathrm{N}$ (picocoulomb per Newton), the charge developed would be about $1000 \mathrm{pC}$ for an axial force of about $500 \mathrm{~N}$. We measured the charge using an Endevco model 2735 charge amplifier. This instrument accepts a maximum charge of $2000 \mathrm{pC}$ and has a useful dynamic range of $30,000: 1$, well suited to our experiment. 
For data acquisition we used a microcomputer set up for two channel analogue-to-digital conversion. The strain gauge incorporated in a lead-compensated Wheatstone bridge and buffered by an instrumentation amplifier, provides one channel, the charge amplifier provides the other. The closing of a foil switch at the hammer point initiated digitization.

\section{(b) Analytical procedure and results}

Samples were collected from a $1.5 \mathrm{~m}$ thick, pure quartz vein which belongs to a large parallel vein system emerging in a metamorphic and plutonic complex in the Fairview mining area of British Columbia, Canada (Okulitch 1969, 1977; Parkinson, 1985).

Several thin-sections prepared from a large specimen showed a heterogeneous grain-size ranging from $0.2-1 \mathrm{~mm}$ (average $0.5 \mathrm{~mm}$ ) for about $80 \%$ of the inspected surface. Fine grains $(<0.2 \mathrm{~mm})$ cover about $10 \%$ of the surface. The large grains $(>1 \mathrm{~mm})$ demonstrate a strong undulatory extinction.

As a preliminary test for presence of a piezoelectric fabric, three cubic samples $(2.5 \times 2.5 \times 2.5 \mathrm{~cm})$ were cut from a large rock specimen $(7 \times 10 \times 15 \mathrm{~cm})$. The measured piezoelectric coefficient along one of the axes of the samples was as high as $1.5 \%$ of single crystal (about 5 times the expected statistical effect). The similar faces of the three samples demonstrated the same polarity while the magnitude of the polarization varied by $50 \%$ among the three samples. This reproducibility of the signal from sample to sample is indicative of a fabric related effect (Bishop, 1981b; Barid and Kennan, 1985). In order to explore the orientation of maximum piezoelectricity, a spherical sample, $21 \mathrm{~mm}$ in diameter, was shaped from a fractureless part of the large specimen.

The orientations of measurement were marked by about 150 points on one hemisphere, with approximately equal-area spacing.

The sample was held between two concave electrodes covering about 4 degrees around the pole of measurement. The optimal size of the electrodes for monitoring nearly all of the volume polarization depends primarily on the thickness of the sample and its dielectric constant. Experiments on cubic samples showed that, for a $2 \mathrm{~cm}$ thick sample, the size of the electrodes can be reduced to $0.5 \mathrm{~cm}^{2}$ without significant loss in signal level. 


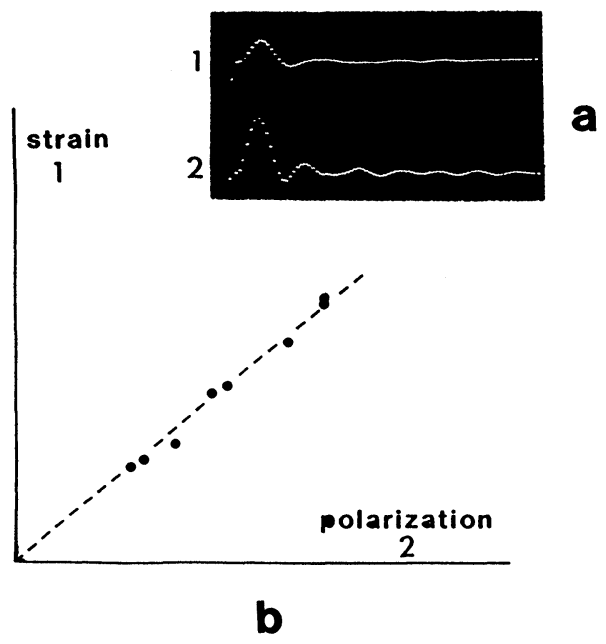

Figure 2 (a) Typical digitized signals from the strain gauge and the charge amplifier. (b) Relative piezoelectric response to different impulses, measured on the spherical sample. The measurements correspond to the amplitude of output signals from the charge amplifier and the strain gauge (a test of proportionality).

Several tests were carried out at each point with application of different amounts of mechanical force. The ratio of the developed charge to the applied force (amplitude of the signal from the strain gauge) was constant (Figure 2). This proportionality is evidence that the measured effect is associated with piezoelectricity. Another important characteristic of the piezoelectric effect is the reversal of polarity with 180 degree rotation of the sample. This characteristic was also consistently observed both on cubic and spherical samples.

The apparatus was unloaded after completion of a set of measurements at each point. The next point was then aligned with the pressure axis and the system was pretensioned again with the same static force. The whole measurement procedure (alignment, static loading and application of dynamic force) was repeated for several points after a few days. The results were reproducible within 10 to $20 \%$ respectively for large and average signals. The points with small signals (less than $0.5 \%$ of a single crystal) were poorly reproducible, within $20 \%$ to $50 \%$. The inevitable small error in 

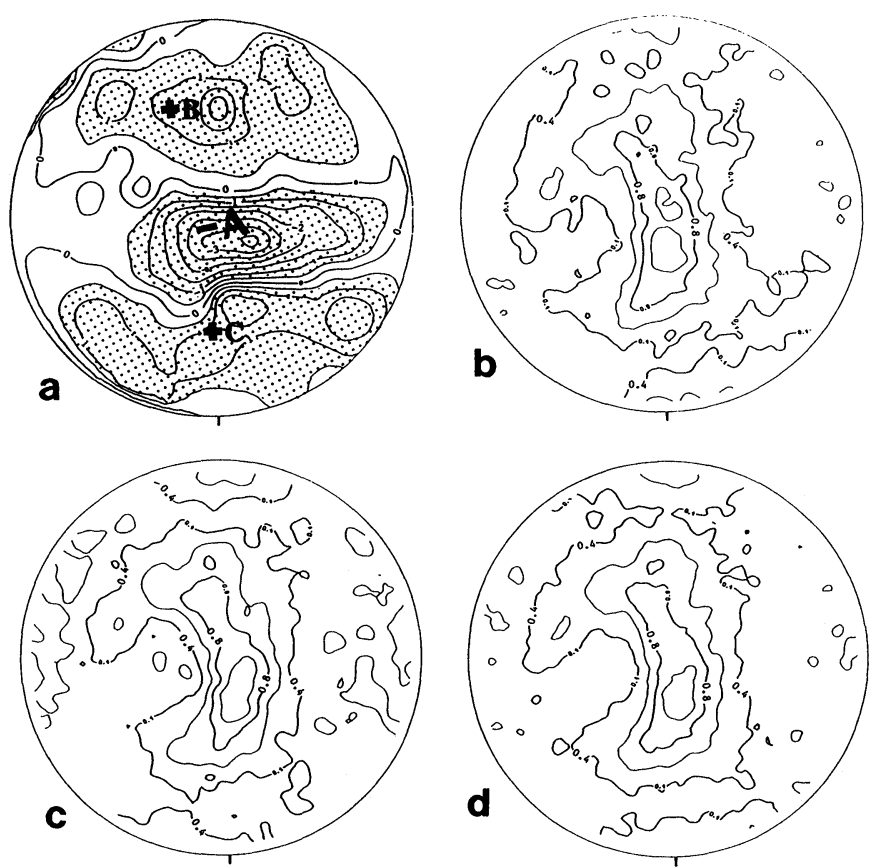

Figure 3 (a) Contoured equal-area projection of longitudinal piezoelectric coefficients measured along 150 orientations of a spherical sample. The unity contour corresponds to $0.035 \mathrm{pC} / \mathrm{N}$ (about $1.5 \%$ of the longitudinal piezoelctric modulus of a perfect single crystal. (b) \& (d) $a$-axes pole figures from neutron diffraction data for two separate runs (I \& II respectively) with different grids (test of reproducibility). (c) The final $a$-axes pole figures based on combination of the data from the runs I and II. In (b), (c) and (d) the contours are defined by (Signal-Background)/Background. The contour line 0.4 is approximately the expected signal $(0.38 \pm 0.05)$ for a random distribution.

alignment is one of the sources of poor repeatablity for small signals.

A contoured equal-area projection of the data on the lower hemisphere is shown in Figure 3a.

\section{NEUTRON DIFFRACTION ANALYSIS}

Fabric related piezoelectricity should correlate with preferred orientation of the $a$-axes in the aggregate (Wellmer, 1970; Bishop, 
1981a). Neutron diffraction analysis of spherical samples (Wenk et al. 1984; Wenk, 1985) has proven to be efficient in the case of coarse-grained aggregates where the traditional X-ray analysis may suffer from poor statistics. Note that X-ray diffraction observes only the surface grains over a limited area of about 1 to $2 \mathrm{~cm}^{2}$. Neutron diffraction, like piezoelectricity, is a body effect and observes all the grains within the volume of a relatively large sample. This provides an interesting ground for investigating the correlation between the piezoelectric effect and the crystallographic fabric, as it is possible to observe the same volume of material by neutron diffraction and piezoelectric measurements.

Neutron diffraction data were collected on the double axis neutron diffractometer (Figure 4), at the TRIUMF Thermal Neutron Facility (TNF). Neutrons from the TNF are monochromated by a $6 \mathrm{~cm}$ thick stack of $10 \mathrm{~cm}$ diameter $\mathrm{Si}$ wafers which are oriented to reflect from the [422] planes at 90 degrees from the incoming beam. The wave length of the neutrons is then $1.58 \AA$, nearly the same as for $\mathrm{Cu} \mathrm{K}_{\alpha} \mathrm{X}$-rays $(1.54 \AA)$. The neutron flux at the sample is $3-6 \times 10^{4} \mathrm{n} \mathrm{cm}^{-2} \mathrm{~s}^{-1}$ and is nearly uniform over the beam cross-section, approximately $10 \mathrm{~cm} \times 10 \mathrm{~cm}$. The number of neutrons incident on the sample is monitored by a fixed stack of $\mathrm{Si}$ wafers which are oriented to reflect in the parallel position into a fixed detector.

The neutrons reflected from the sample are collected by a bank of four $15 \mathrm{~cm} \times 2.5 \mathrm{~cm}^{3} \mathrm{He}$ detectors located $162 \mathrm{~cm}$ from the sample (Figure 4). The effective resolution of each detector is then $0.80 \times 5.6$ degrees. Two of the detectors are used to measure the $a$-axes ( 110 peaks) while the other two detectors monitor the background.

The sample is mounted on a very thin, annealed $\mathrm{Al}$ holder. The sample holder is attached to a goniometer which is mounted on a motor $(\Phi)$ which can rotate 360 degrees. This entire assembly is mounted on another motor $(\theta)$ whose axis is 90 degrees from the first motor.

The beam size is large enough to allow complete bathing of a relatively large sample. This improves the statistics and compensates to some extent for the low intensity of the beam (when compared with the high flux neutron diffraction facilities, e.g. ILL at Grenoble). 
a
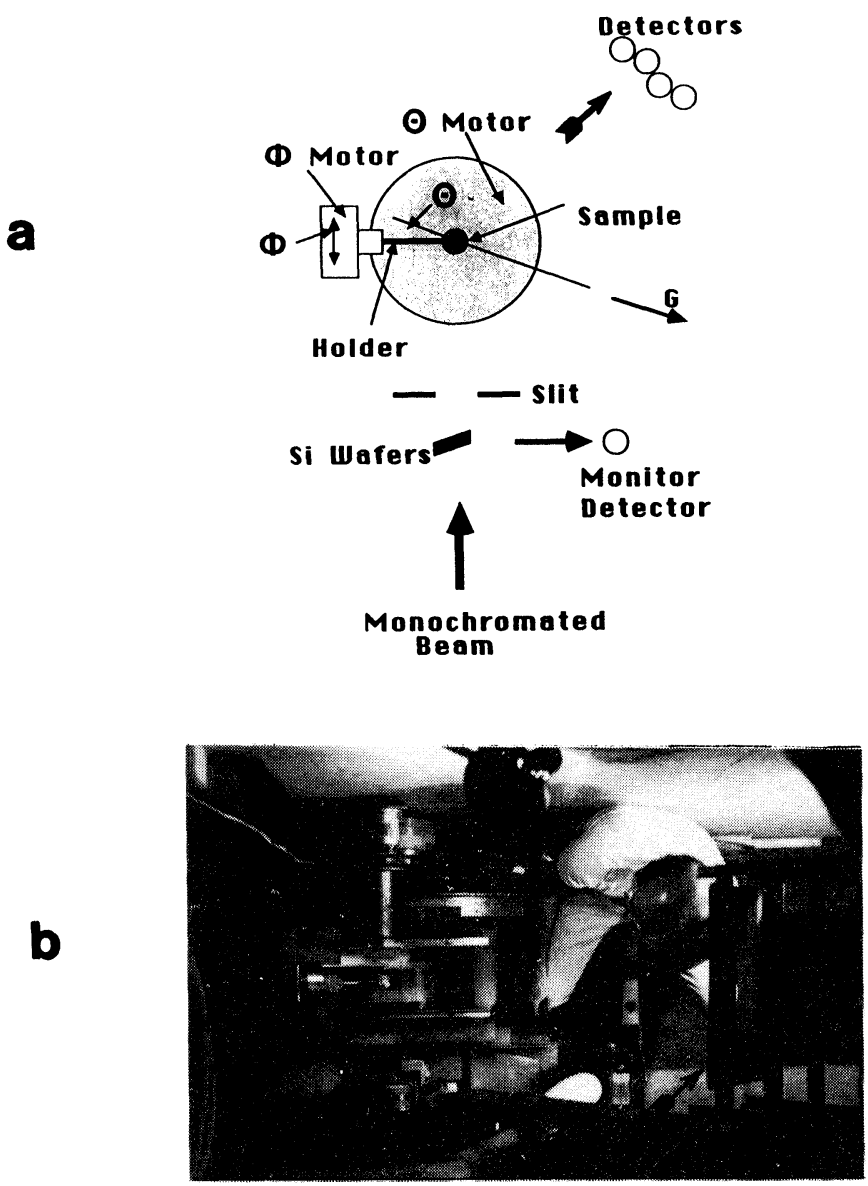

Figure 4 The geometry of the neutron diffraction analysis. (a) side view. (b) Top view; the arrow shows the location of the detectors.

The measured attenuation coefficient was $0.13 \mathrm{~cm}^{-1}$ for our standard quartz crystal and $0.29 \mathrm{~cm}^{-1}$ for the sample under investigation. The attenuation is primarily due to diffuse incoherent scattering and, in a spherical sample, is independent of sample orientation. Considering the low attenuation, all of the grains in the volume of the sample contribute nearly equally to the diffraction data. 
The $a$-axes data were collected in two separate scans, "I" and "II", taken two weeks apart. The data for the two scans were collected on different, nearly uniform grids to $\theta=85$ degrees and with the grid spacing varing from 3 to 5 degrees. Data collection time was 60 to 70 seconds per point for 972 and 1335 points in runs "I" and "II" respectively. In order to test the repeatablity of the results for the adopted data collection technique, the data from the two runs were reduced to pole figures. The results were almost identical (Figures $3 b$ and $3 d$ ). In Figure $3 c$ the data from the two runs are combined.

\section{DISCUSSION}

Assuming an average grain size of $0.5 \mathrm{~mm}$, statistical fluctuations $\left(d_{11} N^{-1 / 2}\right)$ for the spherical sample would be about $0.5 \%$ of piezoelectric effect from a single crystal. However, the effect of large uncompensated grains may produce local effects considerably larger than the statistical effect.

The maximum effect measured on the sphere was more than $7 \%$ of $d_{11}$ for a single crystal. This value is about 15 times larger than the expected statistical effect for the spherical sample.

One of the immediate and important observations on the obtained piezoelectric pole figure is the clear separation of large negative and positive zones. This observation supports the concept of a piezoelectric fabric. Statistical effect and the effect of large uncompensated grains (Tuck et al. 1979) may cause isolated peaks and would appear as details on the larger patterns. The dominance of the fabric related effect is well demonstrated by the presence of a strong piezoelectric effect over a relatively large area of the projection sphere as defined by the major dipole (A). The two minor dipoles (B and C) also demonstrate an overall fabric effect.

Further evidence for the existence of a piezoelectric fabric is the correlation of piezoelectric data with the crystallographic preferred orientation. A well defined preferred orientation is clearly observable on the $a$-axes pole figure obtained by neutron diffraction (Figure 3c). For the purposes of our approximation, the location of the $a$-axes maximum coincides with the location of the maximum piezoelectric effect (in the dipole A). The two minor piezoelectric 
dipoles (B and C) do not correlate with any distinct maxima on the $a$-axes pole figure. It should be noted that neutron diffraction cannot distinguish between positive and negative poles of the $a$-axes. It is therefore possible that the anomalies $\mathrm{B}$ and $\mathrm{C}$ on the piezoelectric pole figure are correlated with the lateral extensions (shoulders) of the major maximum on the $a$-axes pole figure.

The combination of piezoelectric and neutron data demonstrates that the dominant subfabric is a single polar $a$-axes maximum. It is theoretically possible to generate such a fabric with equal percent of the two enantiomorphs (Parkhomenko, 1971). Considering also the minor piezoelectric anomalies, a second subfabric can be assumed with three polar axes (approaching, with some angular discrepancies, the symmetry 32 of a single crystal). Such a fabric cannot be produced from an aggregate with equal percent of the two hands (Parkhomenko. 1971). It is therefore possible that the two hands are present in unequal proportions and the grains of "excess" enantiomorph be responsible for a minor subfabric with three piezoelectric dipoles (Bishop, 1981).

The presence of a piezoelectric fabric was also demonstrated by the consistency of data obtained from several parallel cubic samples cut from a large specimen which favours the concept of continuity of the piezoelectric fabric at a scale larger than the studied spherical sample.

\section{CONCLUDING REMARKS}

Scanning of a spherical sample for the piezoelectric effect has provided purely experimental information about the existence and nature of fabric related piezoelectricity in a coarse-grained quartz aggregate.

The fabric demonstrates a major piezoelectric dipole whose orientation falls on the $a$-axes major maximum.

The piezoelectric effect has applications as an independent or supplementry technique in fabric analysis. It can provide reliable data for coarse-grained quartz aggregates where the traditional $\mathrm{X}$-ray techniques may suffer from poor statistics.

The polar nature of piezoelectricity provides information about 
polarity of the fabric (lack of centre of symmetry). This information, which cannot be obtained from neutron or X-ray diffraction, can complement the diffraction data for a complete solution of the orientation distribution function.

\section{Acknowledgement}

We wish to thank Dr. H. R. Wenk for fruitful discussions on the subject and constructive suggestions for improvement of an early draft of our manuscript. Thanks are also due to Dr. J. G. Souther for reviewing an early manuscript.

The work was funded by research grants from the Natural Sciences and Engineering Research Council of Canada (A-720, CRD8503).

\section{References}

Barid, G. A. and Kennan, P. S. Electrical response of tourmaline rocks to a pressure impulse. Tectonophysics, 111, pp. 147-154 (1985).

Bishop, J. R. Estimating quartz fabrics from piezoelectric measurements. Mathematical Geology, 13, No. 4, pp. 261-289 (1981a).

Bishop, J. R. Piezoelectric effects in quartz-rich rocks. Tectonophysics, 77, pp. 297-321 (1981b).

Cady, W. G. Piezoelectricity (2nd ed.). Dover (2 vols), New York. 1st ed. McGraw-Hill, New York 1946, p. 822 (1964).

Neyshtadt, N. M., Mazanova, V. Z., Binevich, L. Ya. and Maiko, M. I. Piezoelectric method of exploration (methodological recommendations). ONTI VITR, Leningrad (in English translation 53 p) (1972).

Nitsan, U., Electromagnetic emission accompanying fracture of quartz-bearing rocks. Geophys. Res. Lett., 4, pp. 333-336 (1977).

Nye, J. F. Physical properties of crystals. Clarendon Press, Oxford, p. 322 (1957).

Okulitch, A. V. Geology of Mount Kobau. Ph.D. Thesis, University of British Columbia, Canada (1969).

Parkhomenko, E. I. Electrification phenomena in rocks. Translation by G. V. Keller. Plenum Press, New York (1971).

Sobolev, G. A.., Demin, V. M., Narod, B. B. and Whaite, P. Tests of piezoelectric and Pulsed-radio methods for quartz vein and base metal sulphides, prospecting at Giant Yellowknife Mine, N. W. T and Sullivan mine Kimberley, Canada. Geophysics, 49, No. 12, pp. 2178-2185 (1984).

Sobolev, G. A., Demin, V. M., Los, V. F., and Maybuk, Ya. Study of the electromagnetic radiation of rocks containing semiconductor and piezoelectric minerals. (Izv. Earth Physics, Vol. 18, No. 11), Izv. Akad. Nauk, Sci. USSR, Physics of Solid Earth, 18, pp. 888-897) (1982).

Sobelev, G. A., and Demin, V. M. Mechanoelectrical phenomena in the earth. Moscow, Nauka (Partial English translation by G. Volkoff) (1980).

Tuck, G. J., Stacey, F. D., and Starkey, J. A search for the piezoelectric effect in quartz bearing rocks. Tectonophysics, 39, pp. T7-T11 (1977).

Warwick, J. W., Stoker, C. and Meyer, T. R. Radio emission associated with rock fracture: possible application to the Great Chilean earthquake of May 22, 1960. J. Geophys. Res., 87(B4), 2851-2859 (1982).

Wellmer, F. W. Mathematische Auswertung von Piezoelektrischen Messungen in der 
Gefügekunde. Clasthaler Tektonische, Hefte 10, (Computereinsatz in Geologie), pp. 276-292 (1970).

Wellmer, W. F. An approximation method for evaluation of the third rank piezoelectric tensor of the symmetry $3: 2$ and its application in petrofabric studies. Mathematical Geology, 3, No. 4, pp. 375-378 (1971).

Wenk, H. R., Kern, H. Schaefer, W., and Will, G. Comparison of Neutron and $\mathrm{X}$-ray diffraction in texture analysis of deformed carbonate rocks. Journal of Structural Geology, 6, No. 6, pp 687-692 (1984).

Wenk, H. R. Preferred Orientation in Deformed Metals and Rocks: An Introduction to Modern Texture Analysis. Academic Press, Orlando, USA. p. 610 (1985). 